\title{
Rumo a uma Teoria de Domínios da Homotopia
}

\author{
Daniel O. Martínez-Rivillas ${ }^{1}$, Ruy J.G.B. de Queiroz ${ }^{1}$ \\ ${ }^{1}$ Centro de Informatica (CIn) \\ Universidade Federal de Pernambuco (UFPE) \\ Recife, PE - Brasil \\ $\{$ domr, ruy $@$ ecin.ufpe.br
}

\begin{abstract}
Resumo. A Teoria dos Domínios de Dana Scott fornece técnicas gerais para a obtenção de $\lambda$-modelos através da resolução de equações de domínio sobre categorias cartesianas fechadas arbitrárias. A intenção aqui é oferecer uma exposição de forma geral do projeto de generalização da Teoria dos Domínios para uma teoria que denominamos "Teoria de Domínios da Homotopia". O esforço vai no sentido de encontrar um tipo de $\lambda$-modelo com uma estrutura de $\infty$-grupóide, que permitem um salto na interpretação das igualdades do $\lambda$ calculo (ex: $\beta$-igualdade, $\eta$-igualdade etc.) para igualdades de ordem superior na mesma teoria.
\end{abstract}

\begin{abstract}
Scott's Domain Theory provides general techniques for obtaining $\lambda$ models, solving domain equations, raised in arbitrary cartesian closed categories. The intention here is to offer an exposition in general terms of the project to generalize Domain Theory for a theory that we call "Homotopy Domain Theory". The effort goes in the way of finding a type of $\lambda$-models with the structure of $\infty$-groupoid, which allows a jump to the interpretation of equalities of $\lambda$-calculus (e.g. $\beta$-equality, $\eta$-equality etc.) for higher equalities of the same theory.
\end{abstract}

\section{Introdução}

A iniciativa de buscar $\lambda$-modelos [Hindley e Seldin 2008] com estrutura de $\infty$-grupóide surgiu em [Martinez e de Queiroz 2020], onde estudou-se a geometria de qualquer c.p.o (e.g. $D_{\infty}$ ), e constatou-se que a topologia inerente a esses modelos gerava grupos de ordem superior triviais. A partir desse momento, surgiu a necessidade de procurar um tipo de modelo que apresentasse uma rica estrutura geométrica; onde seus grupos fundamentais de ordem superior não entrassem em colapso.

Para cumprir este propósito, o caminho que nos pareceu mais natural foi adaptar a Teoria de Domínios de Dana Scott a uma teoria geral dos domínios, onde categorias cartesianas fechadas são substituídas por $\infty$-categorias cartesianas fechadas, como foi feito em [Martinez e de Queiroz 2021]. Essas $\infty$-categorias são definidas nos conjuntos simpliciais, onde aqueles que têm uma estrutura de $\infty$-grupóide são chamados complexos de Kan [Lurie 2009], os quais estão relacionados com algumas teorias computacionais, como a Teoria de Tipos de Homotopia (HoTT). De modo a garantir a consistência de HoTT, Voevodsky [Kapulkin, Lumsdaine e Voevodsky 2012] (ver [Lumsdaine e Shulman 2020] para tipos indutivos superiores) provou que o HoTT possui um modelo na categoria de complexos de Kan (ver [Program 2013]). 
Em [Martinez e de Queiroz 2020] a $\infty$-categoria Kleisli $K l\left(L_{P}^{*}\right)$ (como uma generalização de [Hyland 2010]) é apresentada como um exemplo de uma $\infty$-categoria cartesiana fechada (c.c.i) com pontos suficientes, onde $L_{P}^{*}$ é a comônada sobre $K l(P)$ que fecha $\infty$-categorias pequenas a $\infty$-categorias que preservam colimites $\kappa$ pequenos, a estrutura Kleisli $P X=\left[X^{o p}, \mathcal{S}\right]$, $\operatorname{com} X$ pequena e $\mathcal{S}$ a $\infty$-categoria dos espaços, e $K l(P)$ a $\infty$-categoria Kleisli gerada pela estrutura $P$. Finalmente em [Martinez e de Queiroz 2021] são aplicados os métodos generalizados para resolução de equações de domínio em c.c.i (que chamamos "Equações de Domínio da Homotopia”) ao caso particular $K l\left(L_{P}^{*}\right)$, onde alguns complexos de Kan (com informação relevante) são apresentados como exemplos de soluções deste tipo de equações.

\section{Equação de Domínio da Homotopia em uma $\infty$-categoria cartesiana fechada arbitrária}

Esta seção é uma generalização direta dos métodos tradicionais para resolver equações de domínio em categorias cartesianas fechadas (ver [Asperti e Longo 1991] e [Abramsky e Jung 1994]), no sentido de obter complexos de Kan como soluções de um tipo de equações, que chamamos Equações de Domínio de Homotopia, em qualquer $\infty$ categoria cartesiana fechada (cf. [Martinez e de Queiroz 2021]). A seguir, temos uma versão $\infty$-categórica do teorema do ponto fixo.

Teorema 2.1. Seja $\mathcal{K}$ uma $\infty$-categoria. Seja também $F: \mathcal{K} \rightarrow \mathcal{K}$ um funtor (covariante) $\omega$-contínuo e tome um vértice $K_{0} \in \mathcal{K}$ tal que existe uma aresta $\delta \in \mathcal{K}\left(K_{0}, F K_{0}\right)$. Assuma também que $\left(K,\left\{\delta_{i, \omega} \in \mathcal{K}\left(F^{i} K_{0}, K\right)\right\}_{i \in \omega}\right)$ é um colimite para o $\omega$-diagrama $\left(\left\{F^{i} K_{0}\right\}_{i \in \omega},\left\{F^{i} \delta\right\}_{i \in \omega}\right)$, onde $F^{0} K_{0}=K_{0} e F^{0} \delta=\delta$. Então $K \simeq F K$.

Para aplicar o Teorema 2.1 na equação do domínio da homotopia $X \simeq(X \Rightarrow$ $X)=F X$ em uma $\infty$-categoria cartesiana fechada $\mathcal{K}$, primeiro precisamos garantir a continuidade de $F: \mathcal{K} \rightarrow \mathcal{K}$. Para isso, o problema do funtor contravariante do exponencial é resolvido de forma semelhante ao caso tradicional [Asperti e Longo 1991]. Ou seja, uma $\infty$-categoria $K^{H P r j}$ é definida, com os mesmos objetos de $K$, e as projeções fracas (que chamamos "projeções de homotopia") como seus morfismos. Isto com o fim de transformar um funtor contravariante (como o funtor exponencial) em um funtor covariante, conforme definido abaixo (a definição de $(0, \infty)$-categoria pode ser consultada en [Martinez e de Queiroz 2021]).

Definição 2.1. Dada uma $(0, \infty)$-categoria $\mathcal{K}$, e um funtor contravariante no primeiro componente $F: \mathcal{K}^{o p} \times \mathcal{K} \rightarrow \mathcal{K}$, o funtor covariante $F^{+-}: \mathcal{K}^{H P r j} \times \mathcal{K}^{H P r j} \rightarrow \mathcal{K}^{H P r j}$ é definido por

$$
\begin{aligned}
& F^{+-}(A, B)=F(A, B), \\
& F^{+-}\left(\left(f^{+}, f^{-}\right),\left(g^{+}, g^{-}\right)\right)=\left(F\left(f^{-}, g^{+}\right), F\left(f^{+}, g^{-}\right)\right),
\end{aligned}
$$

onde $A, B$ são vértices, $e\left(f^{+}, f^{-}\right),\left(g^{+}, g^{-}\right)$são pares $n$-simplexos em $\mathcal{K}^{H P r j}$.

Observação 2.1. Seja $\mathcal{K}$ uma $(0, \infty)$-categoria cartesiana fechada, $\omega^{o p}$-completa. Já que o funtor exponencial $\Rightarrow: \mathcal{K}^{o p} \times \mathcal{K} \rightarrow \mathcal{K}$ e o funtor diagonal $\Delta: \mathcal{K} \rightarrow \mathcal{K} \times \mathcal{K}$ são localmente contínuos, os funtores associados

$$
(\Rightarrow)^{+-}: \mathcal{K}^{H P r j} \times \mathcal{K}^{H P r j} \rightarrow \mathcal{K}^{H P r j}, \quad(\Delta)^{+-}: \mathcal{K}^{H P r j} \rightarrow \mathcal{K}^{H P r j} \times \mathcal{K}^{H P r j}
$$


são $\omega$-contínuos. Mas a composição de funtores $\omega$-contínuos ainda é um funtor $\omega$ continuo. Portanto, o funtor

$$
F=(\Rightarrow)^{+-} \cdot(\Delta)^{+-}: \mathcal{K}^{H P r j} \rightarrow \mathcal{K}^{H P r j},
$$

é $\omega$-contínuo. Pelo Teorema 2.1 o funtor $F$ tem um ponto fixo, isto é, existe um vértice $K \in \mathcal{K}$ tal que $K \simeq(K \Rightarrow K)$. A $\infty$-categoria dos pontos fixos do $F$ é denotada por $\operatorname{Fix}(F)$.

\section{Equação de Domínio da Homotopia em $K l\left(L_{P}^{*}\right)$}

Em [Martinez e de Queiroz 2020] provou-se que as $\infty$-categorias $K l\left(L_{P}^{*}\right)$ e $K l(P)$ são cartesianas fechadas. Portanto, podemos aplicar a teoria dos domínios de homotopia da seção anterior. Para um cardinal regular $\kappa$, seja a subcategoria $\mathcal{P} r_{\kappa}^{L}$ de $C A T_{\infty}$, cujos objetos são $\infty$-categorias $\kappa$-compactamente geradas e cujos morfismos são funtores que preservam colimites pequenos e objetos $\kappa$-compactos. De acordo com a imersão $\operatorname{Pr}_{\kappa}^{L}(P A, P B) \hookrightarrow K l(P)(A, B)$ temos o seguinte resultado.

Proposição 3.1. A $\infty$-categoria $K l(P)$ admite limites para $\omega^{o p}$-diagramas em $\mathcal{P} r_{\kappa}^{L}$. Logo, $K l\left(L_{P}^{*}\right)$ também admite este tipo de limites.

A seguinte proposição garante que existem objetos reflexivos que não são equivalentes ao complexo de Kan trivial $\triangle^{0}$. Sua prova pode ser encontrada em [Martinez e de Queiroz 2021].

Proposição 3.2. Existe um objeto reflexivo não contratível $K l\left(L_{P}^{*}\right)$.

O fato de que um complexo de Kan $X$ é não contratível, não implica que cada vértice $x \in X$ contém informação, ou que este contém buracos em todas as dimensões superiores. Isto motiva a seguinte definição.

Definição 3.1 (Complexo de Kan fragmentado). Um complexo de Kan pequeno X é fragmentado se

1. $\pi_{0}(X)$ é infinito.

2. para cada $n \geq 1$, existe um vértice $x \in X$ tal que $\pi_{n}(X, x) ¥ *$.

3. para cada vértice $x$ de algum $k$-simplex não trivial $X_{k}$ (i.e., nenhuma aresta de $X_{\kappa}$ é a identidade) contido em $X$, com $k \geq 2$, existe $n \geq 1$ tal que $\pi_{n}(X, x) ¥ *$.

Exemplo 3.1. Para cada $n \geq 0$, seja o complexo de Kan $B^{n} \cong \partial \triangle_{\bullet}^{n}$ (isomorfos). Onde $\triangle_{\bullet}^{n}$ tem os mesmos vértices e lados de $\triangle^{n}$ mas 1-simplexos inversíveis. E tome o complexo de Kan $B^{\omega}$, com $\omega$ vértices diferentes $B_{0}^{\omega}, B_{1}^{\omega}, B_{2}^{\omega}, \ldots$, tal que existe um mapa $f: \partial \triangle^{n} \rightarrow$ $B^{\omega}$, com $f(i)=B_{i}^{n}$ para cada $0 \leq i \leq n$, o qual estabelece $f \partial \triangle \cong B^{n}$. Defina o complexo de Kan fragmentado $B_{0}$ como a união disjunta $B_{0}=\coprod_{n \leq \omega} B^{n}$.

Note que $B^{n}$ é "similar"à esfera $S^{n-1}$. Além do mais, $\pi_{n-1}\left(B^{n}\right) \nsucceq *$ para todo $n \geq 2$, e existe $k \geq n$ tal que $\pi_{k}\left(B^{n}\right) \not *$ para cada $n \geq 3$ [Hatcher 2001]. Para mais exemplos ver [Martinez e de Queiroz 2021].

Definição 3.2 ( $\lambda$-modelo de homotopia). Seja $\mathcal{K} \hookrightarrow C A T_{\infty}$ uma $\infty$-categoria cartesiana fechada com uma quantidade suficiente de pontos. Um complexo de Kan $K \in \mathcal{K}$ é um $\lambda$-modelo de homotopia se $K$ é um complexo de Kan fragmentado e reflexivo. 
Exemplo 3.2. Dado o complexo de Kan fragmentado $B_{0}$ do Exemplo 3.1. Partindo da projeção diagonal, $B_{0} \stackrel{\delta_{0}}{\longrightarrow}\left(B_{0} \Rightarrow B_{0}\right)$, o objeto inicial $B_{0}$, irá gerar o complexo de Kan fragmentado $B$ in Fix $(F)$ como o colimite do $\omega$-diagrama $\left(\left\{F^{i} B_{0}\right\}_{i \in \omega},\left\{F^{i}\left(\delta_{0}, \gamma_{0}\right)\right\}_{i \in \omega}\right)$, onde $\gamma_{0}:\left(B_{0} \Rightarrow B_{0}\right) \rightarrow B_{0}$ é o membro direito do par projeção.

\section{Conclusões e trabalhos futuros}

Alguns métodos foram estabelecidos para resolver equações de domínio de homotopia, o que contribui ainda mais para o projeto de uma generalização da Teoria de Domínios para uma Teoria de Domínios da Homotopia (HoDT). Além disso, usando esses métodos de resolução de equações, foi possível obter alguns modelos de homotopia específicos em uma $\infty$-categoria cartesiana fechada. Isto poderia ajudar a definir uma teoria geral de $\lambda$-calculo de ordem superior. Para trabalhos futuros, seria interessante ver qual for a relação entre a teoria de um $\lambda$-modelo de homotopia e a Teoria dos Tipos da Homotopia (HoTT).

\section{Referências}

Abramsky S. and Jung A. (1994). Domain theory. In S. Abramsky, D. M. Gabbay, and T. S. E. Maibaum, editors, Handbook of Logic in Computer Science, volume 3, pages 1-168, Clarendon Press.

Asperti A. and Longo G. (1991). Categories, Types and Structures: An Introduction to Category Theory for the working computer scientist. Foundations of Computing Series, M.I.T Press.

Hatcher A. (2001) Algebraic Topology. Cambridge University Press, New York, NY.

Hindley J. and Seldin J. (2008). Lambda-Calculus and Combinators, an Introduction. Cambridge University Press, New York.

Hyland M. (2010) Some reasons for generalizing domain theory. Mathematical Structures in Computer Science 20, pages 239-265.

Kapulkin C., Lumsdaine P. and Voevodsky V. (2012). The simplicial model of univalent foundations. arXiv:1211.2851.

Lumsdaine P. and Shulman M. (2020). Semantics of higher inductive types. Mathematical Proceedings of the Cambridge Philosophical Society 169, pages 159-208.

Lurie J. (2009). Higher Topos Theory. Princeton University Press, Princeton and Oxford.

Martínez-Rivillas D. and de Queiroz R. (2021). The $\infty$-groupoid generated by an arbitrary topological $\lambda$-model. Logic Journal of the IGPL. https://doi.org/10.1093/jigpal/jzab015 (also arXiv:1906.05729).

Martínez-Rivillas D. and de Queiroz R. (2020). Towards a homotopy domain theory. arXiv:2007.15082.

Martínez-Rivillas D. and de Queiroz R. (2021). Solving homotopy domain equations. arXiv:2104.01195.

Program T. U. F. (2013). Homotopy Type Theory: Univalent Foundations of Mathematics. Princeton, NJ: Institute for Advanced Study. 